\title{
NARRATIVAS TECNOLÓGICAS: UN EJEMPLO DE APLICACIÓN DE LA SOCIOLOGÍA DE LAS ASOCIACIONES
}

\section{TECHNOLOGICAL NARRATIVES: AN APPLIED EXAMPLE OF THE SOCIOLOGY OF ASSOCIATIONS}

Mg. Verónica Devenin (vdevenin@gmail.com) Departamento de Sociología y Antropología, Universidad de Concepción (Concepción, Chile).

Mg. Guillermo Henríquez (ghenriqu@udec.cl) Departamento de Sociología y Antropología, Universidad de Concepción (Concepción, Chile).

\begin{abstract}
Are social explanations, as we know them, adequate to account for the complexity of contemporary society and the ongoing emergence of new actors and new phenomena? To the Actor-Network Theory, there are no doubts: social factors are not sufficient to explain the dynamics of society. It is necessary to use heterogeneous factors, taking into account non-human elements and technology. That is, to explain the social, it's necessary to consider social relations, "non-human actants" and the technical procedure in which they are involved. This article will present the conceptual foundations of this new sociological approach, an applied example in the context of research conducted on innovation, and, finally, some lessons and conclusions regarding their applications, potentials and limitations.
\end{abstract}

Key words: actor-network theory, sociology of associations, methodology, technological narratives, innovation.

\section{Resumen}

¿Son las explicaciones sociales, tal como las conocemos, adecuadas para dar cuenta de la complejidad de nuestra sociedad actual y de la irrupción permanente de nuevos actores y nuevos fenómenos? Para la Teoría del Actor-Red la respuesta es taxativa: los factores sociales no son suficientes para explicar la dinámica de la sociedad, es preciso recurrir a medios heterogéneos, tomar en consideración lo no humano, recurrir a lo tecnológico. Es decir, para explicar lo social es necesario considerar tanto las relaciones sociales, a los "actantes no humanos" y los procedimiento técnicos en los que están involucrados. El presente artículo presentará las bases conceptuales de esta nueva aproximación sociológica, un ejemplo de aplicación en el marco de una investigación desarrollada en materia de innovación, para finalmente terminar con algunos aprendizajes y conclusiones respecto de sus usos, potencialidades y limitaciones.

Palabras clave: teoría del actor-red, sociología de las asociaciones, sociología de lo social, narrativas tecnológicas, innovación. 


\section{Introducción}

Para la Teoría del Actor-Red (1), el objetivo de la sociología no es el de entregar explicaciones sociales donde emergen grandes categorías o fuerzas sociales que actúan misteriosamente como causas que pueden explicar demasiados efectos. Ni tampoco concentrarse en las interacciones localizadas cara a cara, dando un lugar privilegiado a las explicaciones de nivel micro. La sociología requiere renovarse para poder dar cuenta de los fenómenos y actores emergentes, develando relaciones más robustas y descubriendo patrones más reveladores, a través del rastreo -y registro- de asociaciones entre entidades que son de distinta naturaleza, no sólo de materia social. La propuesta de esta teoría es resignificar la disciplina, pasando desde lo que ellos llaman una sociología de lo social a una sociología de las asociaciones.

Comenzado por entender de forma distinta qué es lo social, en adelante expondremos los conceptos más relevantes de esta nueva sociología y sus diferencias con la sociología tradicional, y exploraremos una aplicación concreta de sus postulados para poder dar cuerpo a su enfoque. Esperamos que esta exposición y discusión contribuya a la consideración de la sociología de las asociaciones como una nueva alternativa epistemológica, y en un plano más concreto, como una nueva aproximación metodológica.

\section{La Sociología de las Asociaciones}

El punto de partida de la bifurcación entre la sociología tradicional y la sociología de las asociaciones es qué se entiende por lo social.

Tanto en el contexto de la disciplina, así como de forma extendida en el sentido común, lo social ha remitido y sigue remitiendo a un determinado orden de cosas, algo así como un tipo de material, un tipo específico de fenómeno. Se trataría de un dominio de la realidad que se puede distinguir de otros tales como la economía, la geografía, la biología, el derecho, etc. Sin importar lo vago que pueda ser su definición, una vez establecido su dominio, éste se puede utilizar para dar cuenta de fenómenos "específicamente" sociales y aportar un cierto tipo de explicación para aquello de lo que no pudieran dar cuenta los otros dominios: los “aspectos sociales" de fenómenos no sociales (Latour 2008:15).

Para la sociología de las asociaciones, lo social no es ni un tipo de dominio, ni de contexto, ni de fuerza.

Cuántas veces nos ha ocurrido que frente a un objeto de estudio, la disección que se realiza entre lo que se entiende por social y aspectos pertenecientes a otros dominios nos aparece como forzado. Cuando se divide cualquier estado de cosas en un componente material al que se agrega como apéndice uno social, lo que ocurre es una imposición de disputas disciplinarias, no es la imposición de un requisito empírico (Latour 2008:123).

La confusión se fue desarrollando en el curso de la historia de la disciplina. Gabriel Tarde, una de las más importantes influencias de esta aproximación, señalaba ante la posición de cada vez mayor hegemonía que alcanzaba Emile Durkheim, que éste había abandonado la tarea de explicar la sociedad, confundiendo causa y efecto, remplazando la comprensión del vínculo social con un proyecto político que apuntaba a la ingeniería social. Para Tarde, lo social debía considerarse como un fluido circulante que debía seguirse con nuevos métodos y no como un tipo de organismo nuevo (2). La pregunta que debe responder la sociología es cómo se sostiene unida la sociedad, envés de utilizar la sociedad para explicar otra cosa (Latour 2008:30). 
Retomando a Tarde, la sociología de las asociaciones considera que lo social no está en lugar alguno en particular, como una cosa entre otras cosas, sino que puede circular como un movimiento que relaciona cosas no sociales: lo social es la asociación (Latour 2008:156).

En este nuevo paradigma, los objetos adquieren capacidad de agencia. Esto porque, para la sociología de lo social, la "sociedad", las "normas sociales", las "leyes", las "estructuras", los "hábitos" o las "reglas" no tendrían suficiente fortaleza para explicar la durabilidad de los vínculos, particularmente las asimetrías y las desigualdades. La existencia de vínculos duraderos no prueba que estos estén hechos de materia social.

El vínculo social tiene muchas dificultades para extenderse en el tiempo y en el espacio, no tiene inercia y debe ser renegociado permanentemente. Para establecer relaciones de poder o asimetrías de modo duradero, es necesario convertir los vínculos débiles y de rápida desaparición en otro tipo de vínculos (Latour 2008:100). La influencia de Michel Foucault se hace explícita en este planteamiento: mecanismos, aparatos, maquinarias son nociones que emergen como elementos activos, tanto como la agencia humana, en la construcción de explicaciones (Domènech y Tirado 1998).

Por esto no se debe entender una simetría absurda entre humanos y no humanos. El concepto de simetría utilizado por la sociología de las asociaciones significa no imponer a priori una asimetría espuria entre la acción humana intencional y un mundo material de relaciones causales (Latour 2008:113). Es decir, no establecer ninguna clasificación a priori que diferencie entre lo social y lo natural o lo natural y lo tecnológico, y evitar hacer prevalecer una entidad por sobre otra, considerando las relaciones entre humanos como más relevantes que las que se dan entre humanos y no humanos, o incluso entre no humanos (Domènech y Tirado 1998:25).

Que los objetos también puedan ser agencias es posible por cuanto pueden constituirse como mediadores.

Por mediadores se entiende algo o alguien que transforma, traduce, distorsiona y modifica el significado o los elementos que se supone deben transportar. Las cosas pueden autorizar, permitir, dar recursos, alentar, sugerir, influir, bloquear, hacer posible, prohibir, etc. Lo "no humano" debe ser considerado como un "actante" más, entendiendo por ello cualquier entidad que actúa en una trama, sea cual fuere el papel que se le atribuye (Latour 1998b:255).

Los mediadores se diferencian de los intermediarios, entendiendo por estos últimos personas u objetos que transportan un significado o fuerza sin transformación, y que por lo tanto, transportan las causas sin modificar sus efectos. En el caso de la sociología de las asociaciones, existen múltiples mediadores, de distinta naturaleza, que hacen que las causas no sean más que precedentes, circunstancias u ocasiones.

Una concatenación de mediadores no establece las mismas relaciones y no requiere el mismo tipo de explicaciones que un cortejo de intermediarios que transporta una causa. Estas relaciones que transportan transformación son denominadas traducción (Latour 2008:156).

El concepto de traducción remite al proceso a través del cual aparece una totalidad a partir de todas las partes, es decir, la dinámica que rige los entramados de entidades y materiales heterogéneos. Los efectos de la traducción están condenados a no durar, pues volverán a ser traducidos desde el momento en que se altere el estado de las cosas. Esto puede ser consecuencia de la yuxtaposición con otras entidades, de la conexión azarosa con algún elemento, de la inclusión a la red de una nueva entidad, lo que trae consigo la 
reorganización, la traducción de toda la red, sus entidades y significados. Esta dinámica debe ser entendida entonces como un proceso de transformación perpetua, continua e inaprensible durante largos periodos de tiempo, diferenciándose, a juicio de los adherentes a la sociología simétrica, de los ejercicios de representación, imitación o reflejo más propios de las ciencias sociales (Domènech y Tirado 1998:27).

A la luz de estos conceptos se puede describir más adecuadamente el objetivo de la sociología: "no hay sociedad, dominio de lo social ni vínculos sociales, sino que sólo existen traducciones entre mediadores que pueden generar asociaciones rastreables" (Latour 2008:158).

La sociología de las asociaciones diferenciará entonces "sociedad" como el conjunto de entidades ya ensambladas que los sociólogos de lo social consideran hechas de materia social, de "colectivo", utilizado para designar al proyecto de ensamblar nuevas entidades que hasta ahora no habían sido reunidas y que por tal razón aparecen claramente como no compuestas de materia social (Latour 2008:111).

A continuación se presentan algunas de las diferencias más relevantes entre la sociología de lo social y la sociología de las asociaciones (Latour 2008).

\begin{tabular}{|c|c|}
\hline Sociología de lo social & Sociología de las asociaciones \\
\hline $\begin{array}{l}\text { Lo social es un tipo de material, un dominio de la } \\
\text { realidad. }\end{array}$ & $\begin{array}{l}\text { No existe dimensión social, ni contexto social, ni } \\
\text { fuerzas sociales. Lo social es un movimiento de } \\
\text { asociación y reensamblado. }\end{array}$ \\
\hline $\begin{array}{l}\text { Se tiende a imponer un orden, definiendo } \\
\text { anticipadamente el grupo relevante a analizar. }\end{array}$ & $\begin{array}{l}\text { El "orden" se encuentra después de haber dejado } \\
\text { a los actores desplegar toda la gama de } \\
\text { controversias en las que están inmersos. }\end{array}$ \\
\hline $\begin{array}{l}\text { La acción es apoderada por fuerzas que } \\
\text { confluyen en algún tipo de agencia: sociedad, } \\
\text { cultura, estructura, campos, individuos, etc. }\end{array}$ & $\begin{array}{l}\text { La acción es un nodo. Un actor no sería la fuente } \\
\text { de una acción, sino el blanco móvil de una } \\
\text { enorme cantidad de entidades que convergen } \\
\text { hacia él. }\end{array}$ \\
\hline $\begin{array}{l}\text { Predomina la relación causa-efecto, donde las } \\
\text { causas serían transportadas por intermediarios } \\
\text { que no agregarían nada para concretar su efecto. } \\
\text { De unas pocas causas se podrían deducir todos } \\
\text { los efectos que estaban allí "en potencia". }\end{array}$ & $\begin{array}{l}\text { Las causas no permiten que se deduzcan los } \\
\text { efectos, dado que son simplemente ocasiones, } \\
\text { circunstancias y precedentes. Las causas son } \\
\text { mediadores. En el marco de esta teoría, se trata } \\
\text { de poder remplazar tantas causas como sea } \\
\text { posible por una serie de actores (3). }\end{array}$ \\
\hline $\begin{array}{l}\text { La acción es limitada a lo que los humanos "con } \\
\text { intenciones" y "significado" hacen, excluyendo el }\end{array}$ & $\begin{array}{l}\text { Los objetos pueden estar en el origen mismo de } \\
\text { la actividad social. }\end{array}$ \\
\hline
\end{tabular}




\begin{tabular}{|c|c|}
\hline rol que puedan cumplir los objetos. & \\
\hline $\begin{array}{l}\text { La tarea de explicar comienza luego de haber } \\
\text { introducido una profunda desconfianza respecto } \\
\text { de la existencia misma de los objetos que hay } \\
\text { que explicar: las causas tienen que incluir fuerzas } \\
\text { hechas de sustancia social. }\end{array}$ & $\begin{array}{l}\text { Explicar es un emprendimiento de construcción } \\
\text { de un mundo muy práctico que consiste en } \\
\text { relacionar entidades con otras entidades, es decir } \\
\text { dibujar el trazado de una red. Cada vez que se } \\
\text { dice que A está relacionado con B, es lo social } \\
\text { mismo que se genera. }\end{array}$ \\
\hline $\begin{array}{l}\text { Predominio de la clasificación micro/macro. El } \\
\text { uso de la escala se define como una variable más } \\
\text { a esclarecer antes de iniciar la investigación. }\end{array}$ & $\begin{array}{l}\text { La investigación se debe realizar de forma } \\
\text { "bidimensional". Lo macro no está por encima ni } \\
\text { por debajo de las interacciones sino que } \\
\text { agregado a ellas como otras de sus conexiones. } \\
\text { Lo micro es el punto de llegada de una gran } \\
\text { cantidad de agencias que pululan a su alrededor. }\end{array}$ \\
\hline
\end{tabular}

En cualquier caso, la sociología de las asociaciones no desconoce ni desacredita las aportaciones de la sociología tradicional. Sus conceptos y premisas serían de gran utilidad para empaquetar relaciones sociales que ya son conocidas y actores que son miembros bona fide de la sociedad. No obstante, para descubrir nuevos actores inesperados que han surgido recientemente, o nuevas relaciones sociales, es necesario utilizar un "equipamiento distinto". Latour lo plantea como la diferencia entre aprender a manejar en una carretera ya existente y explorar por primera vez el territorio (Latour 2008:41).

Ese equipamiento está relacionado con el método. Para Latour, el trabajo de rastrear y registrar asociaciones es un trabajo lento que requiere mucha paciencia de los analistas, sin tomar atajos ni salidas fáciles. Con esto se refiere a no acudir a explicaciones pre-fabricadas, marcos conceptuales, categorías o ningún otro elemento que desvíe la atención del rastreo de todos los mediadores, por muy caótico que esto pueda resultar en un determinado momento.

Como una premisa básica, no se debe anteponer cómo están constituidos los agregados sociales antes de empezar la investigación (4). Se debe permitir que los actores desplieguen sus controversias y que se definan en ese transcurso la formación de los grupos relevantes. Tampoco establecer a priori el nivel de análisis -macro o micro - pues desde el punto de vista de esta teoría, los niveles se van confundiendo: lo macro no está por sobre lo micro, sino que se expresa en lo micro; por su parte, lo micro es una expresión de la influencia de muchas entidades.

En relación con el lenguaje, se debe utilizar un repertorio general para evitar confundir las expresiones políticas de los propios actores. La sociología de las asociaciones aboga por un "infralenguaje" que permita el desplazamiento de un marco referencial hacia otro (Latour 2008:50).

Finalmente, lo que debe hacer un buen sociólogo de las asociaciones es escribir informes. Un buen informe es una narrativa, una descripción que rastrea una red, es decir, una serie de acciones donde cada participante es tratado como un mediador. Cada punto del texto podría transformarse en una bifurcación, un evento o el origen de una nueva traducción (Latour 2008:178). 
En esta aproximación, no hay una distinción entre describir y explicar: si una descripción está necesitada de explicación, entonces no es una buena descripción. Lo que se requiere es desplegar plenamente las redes que hacen posible un estado de cosas. Adicionar una explicación sería superfluo (Latour 2008:198).

\section{Una Aplicación: Las Narrativas Tecnológicas}

El principal campo de aplicación de esta nueva aproximación ha sido el de la ciencia, la tecnología y la innovación (5).

Según Michel Callon (1998:143), durante mucho tiempo, los científicos sociales han tratado de explicar el alcance, los efectos y las condiciones de desarrollo de la tecnología, considerándola un objeto específico que presenta un amplio número de problemas que se han intentado resolver usando los distintos métodos al alcance de las ciencias sociales.

Según Latour, la principal dificultad para integrar la tecnología en la teoría social sería la carencia de recurso narrativo: "sabemos cómo describir las relaciones humanas, sabemos cómo describir los mecanismos, frecuentemente intentamos alternar entre texto y contenido para hablar acerca de la influencia que ejerce la tecnología en la sociedad o viceversa, pero todavía no somos expertos en tejer los dos recursos descriptivos en un todo integrado" (1998a:118).

Las narrativas tecnológicas permiten superar esta dificultad.

Una narrativa tecnológica es el registro en un único repertorio del seguimiento de todas las cadenas de asociaciones involucradas en el desarrollo de una innovación.

Por cadenas de asociaciones se debe entender asociaciones de entidades de distinta naturaleza, tanto humanas como no humanas, todas ellas mediadoras. Son éstas y sus transformaciones lo que realmente importa para hacer emerger explicaciones de un fenómeno y por lo tanto es lo que debe ser estudiado.

Cada cadena de asociaciones cuenta con programas de acción y antiprogramas. Todo actante tendría un "programa de acción", entendido usualmente como metas para las entidades humanas y funciones para las no humanas (Latour 1998b:255).

Para poder realizar el registro de la cadena de asociaciones se debe tomar un punto de partida, seleccionar un observador y a partir de su programa de acción describir las cadenas sucesivas de asociaciones. Los antiprogramas se deben entender como los obstáculos que se imponen al programa de acción del actante punto de partida (Latour 1998a:130).

Durante este proceso, el innovador, en cada decisión técnica, prueba las hipótesis sobre las cuales se ha apoyado. Estas hipótesis conciernen tanto a la naturaleza de las entidades que necesita para hacer su proyecto, así como a los deseos, intereses y aspiraciones de dichas entidades, aceptando negociar los contenidos técnicos en el filo de sus pruebas, buscando movilizar más entidades y extender su red (Akrich 2006:180).

Al desplegar una red sociotécnica, definiendo trayectorias mediante la asociación y sustitución sucesiva de actantes, definiendo actantes a través de todas las trayectorias en las que participan, siguiendo las transformaciones $y$, finalmente variando el punto de vista de los observadores, no existe necesidad de 
buscar causalidades adicionales. La explicación emerge una vez que la descripción está saturada (Latour 1998a:139).

Como un ejemplo de narrativa tecnológica, Latour (1998a:118) toma el guión de una trayectoria sociotécnica en torno a la historia de la invención simultánea de la cámara KODAK y del mercado de masas para fotógrafos aficionados, desarrollado por Jenkins, el que presenta la siguiente estructura:

(1) profesional-aficionado (A)/ daguerrotipo (B)

(2) profesional-aficionado (A)/ colodión líquido (C) 1850/fabricación del papel (D) -//- hacérselo todo uno mismo inmediatamente

(3) profesional-aficionado (A)/fabricación del papel (D)/ placas de colodión seco preparadas que se adelantan a su tiempo (E) 1860-1870 -//-

(4) profesional-aficionado/fabricación de papel/ placas de gelatina seca más sensibles 1870-1880/ compañías que fabrican placas que se adelantan a su tiempo -//-

(5) profesional-aficionado/fabricación de papel/placas de gelatina seca/compañías que fabrican placas que se adelantan a su tiempo/máquina de impregnación continua de placas/Eastman -//-

(6) (5)/capital aportado por Strong/COMPAÑÍA EASTMAN DE PLACAS SECAS 1881-1883 -//- bajos precios iniciales/ competencia fácil

(7) (6) consorcio de fabricantes de placas -//- mercado todavía limitado/ placas frágiles

(8) película flexible Walter/cámara de bolsillo Walter 1884 -//-

\section{(..) .........}

(37) (35)/1899 gran industria/producción en masa/mercado de masas se amplía a aficionados de 7 a 77 años de edad/cientos de miles de cámaras vendidas -//-

(fin de la narrativa desarrollada por Jenkins)

Para la lectura y comprensión de la narrativa, se debe considerar que:

- Los números entre paréntesis enumeran las versiones sucesivas de un programa de acción. Los números entre paréntesis en negrilla indica que la cadena de asociación anterior permanece como parte de la nueva versión.

- El doble slash separa los programas de acción de los antiprogramas, dejando los primeros a la izquierda y los segundos a la derecha de la línea divisoria.

- Se establece en ocasiones un referente temporal, no obstante, no es necesario que esté presente en cada nueva versión. En el registro se debe establecer el orden sucesivo de las versiones, pero no se mide regularmente el tiempo. El número y la velocidad de los acontecimientos depende de los movimientos de alianzas o de rupturas de los actores. En la medida que se puede reconstruir tales movimientos, es posible a su vez reconstituir la temporalidad (Latour 1998a:128).

- Si bien la narrativa tiene un final, la innovación no lo tiene, pues permanentemente las cadenas vuelven a transformarse: "una consecuencia importante de registrar de esta forma es que 'la realidad' no es un final, no es un estado definitivo que no requiere ningún esfuerzo. El precio que hay que pagar para mantener la realidad es una continua extensión de la cadena de asociaciones" (Latour 1998a:126).

La narrativa tecnológica puede ser resumida mediante la identificación de cada uno de los programas desde el "observador punto de partida" seleccionado y de cada antiprograma -resistencias al programa- y mediante el registro sucesivo de todos los nuevos actores que emerjan, sean humanos o no humanos, individuales o colectivos. Lo que se debe destacar en este registro es que nunca se está frente a dos 
repertorios -infraestructura y superestructura, técnica y economía, función y estilo-, sino con ensamblajes cambiantes de asociaciones y sustituciones (Latour 1998a:120).

\section{Una Experiencia de Aplicación: Aprendizajes, Potencialidades y Limitaciones}

La metodología de narrativas tecnológicas fue utilizada en una investigación durante el año 2008 acerca de las barreras y facilitadores para la incorporación de tecnología ambiental innovadora en la industria del cobre en Chile. Para ello fueron descritas siete narrativas tecnológicas con los profesionales científicotécnicos de la entidad de investigación y desarrollo que se encontraba desarrollándolas y promoviéndolas. El objetivo de la investigación era identificar factores comunes y diferenciadores en el logro de estabilización de la innovación en el mercado objetivo.

De la experiencia de aplicación de las narrativas tecnológicas

El rastreo de las asociaciones: Para construir la narrativa se debe elegir un observador como punto de partida, el cual traza los programas de acción para el objeto técnico y reacciona para sortear los antiprogramas (Latour 1998a:113). En el caso de la investigación, el observador establecido como punto de partida fue "el innovador", representado por la entidad de investigación y desarrollo. A partir de esta definición, se estableció como unidad de información principal los profesionales científico-técnicos del área ambiental de la entidad de I\&D que participaron directa y activamente en la trayectoria de las ecoinnovaciones estudiadas.

La o las unidades de información son críticas para la construcción de las narrativas. De ello depende la posibilidad de realizar una descripción saturada de la cadena de asociaciones involucrada en el desarrollo de la tecnología. Es posible que una sola fuente de información no sea suficiente para lograr una descripción saturada.

En el caso de esta investigación, una segunda unidad de información fue la documentación de segundo orden: documentos de registro, formales e informales que dieran cuenta de distintos hitos durante la trayectoria de la tecnología. Esta unidad de información fue significativamente relevante en aquellos casos donde el interlocutor no tenía conocimiento pleno del devenir de la tecnología desde sus orígenes.

Se puede decir que la cantidad de unidades de información necesarias y la capacidad de establecer el punto de saturación de la narrativa está relacionada con la magnitud de la innovación así como con el grado de aleatoriedad o deliberación de la innovación.

En el caso de nuestra investigación, las innovaciones no eran nuevas para el mundo: existían en el mercado global, pero constituían novedad para el mercado acotado de la industria del cobre en Chile. En algunos casos requerían de un grado adicional de investigación y desarrollo, en otros requería algunos procesos de adaptación.

La historia de una innovación acotada a un mercado podría eventualmente implicar menos cantidad de tiempo y recursos para la construcción de su narrativa, debido a que el volumen de "entidades" involucradas y su dispersión serían más restringidos en comparación con una innovación que tuviese como contexto el mundo. Entonces, se podría decir que la cantidad de recursos necesarios para construir una narrativa depende del alcance de la innovación de que se trate (6). Lo correcto es plantear esto como una hipótesis ya que en un contexto más acotado, los mediadores igualmente podrían multiplicarse. 
En relación con el grado de aleatoriedad o de deliberación en la innovación, esto también puede tener implicancias significativas, particularmente en términos de las técnicas necesarias para explorar y reconstruir la "historia" de la tecnología así como de las condiciones para determinar la saturación de la descripción.

En el caso de la investigación, el "actor punto de partida" es una organización de I\&D que tiene por objetivo desarrollar y promover innovaciones. En este sentido, existía una estrategia deliberada y, por tanto, un programa de acción consciente de los actores para que la innovación lograse estabilizarse en el mercado. Esto facilitó la construcción de las narrativas pues existía un relativo control y accionar permanente sobre el devenir de la innovación, lo que permitió privilegiar la información de los entrevistados para la construcción y definición de saturación.

No obstante, las innovaciones no siempre son producto de este nivel de deliberación. Toda innovación se puede situar en un continuo, donde en un extremo se encuentran procesos emergentes totalmente no planificados y en el otro procesos estratégica y deliberadamente controlados (Meyer y Schubert 2007:29). Se podría decir entonces que, dependiendo del lugar que la innovación tome en ese continuo, puede depender el tipo de técnicas de recolección de información necesarias, así como también puede variar la forma en que se defina el punto de saturación. En el caso de los procesos más bien emergentes, a diferencia de aquellos donde el proceso es más controlado, la identificación de uno, o unos pocos interlocutores, que detenten un conocimiento vasto sobre el recorrido de la innovación es menos probable, por lo que las entrevistas pueden no ser suficientes para recuperar la trayectoria del objeto técnico ni para establecer el punto de saturación.

Acerca de la negociación del lenguaje: Uno de los aspectos críticos para la construcción de la trayectoria y narrativa de la tecnología es la construcción de un lenguaje común entre entrevistador e interlocutor, considerando que éste último es un profesional científico-técnico.

Laudel y Gläser llegaron a la conclusión que es inevitable la necesidad de aprender ciencia para toda investigación que tenga como informantes a científicos. Cuando el contenido de la ciencia bajo investigación forma parte de la explicación sociológica, no es suficiente comprender la investigación o el trabajo del interlocutor como un contexto social relevante o como un marco de referencia. La investigación científica de este tipo requiere comprender los problemas, estrategias y lógica de la investigación científica, e incluir factores no sociales en nuestras explicaciones (Laudel y Gläser 2007:99).

En efecto, en las narrativas desarrolladas en la investigación se pueden encontrar varios elementos que contienen descripciones técnicas, ya sea con base en la física, química y/o biología. Para lograr inscribir estos elementos fue necesaria una explicación técnica detallada de la tecnología y su desarrollo por parte del interlocutor, de forma de facilitar una mejor comprensión de quien realiza el registro y por ende una mejor interpretación de la información que proveían los interlocutores al momento de construir la trayectoria. Esto dio paso a la construcción espontánea de un lenguaje común que permitía comprenderse mutuamente en la interacción.

Laudel y Gläser identificaron tres tareas claves para realizar entrevistas informadas a científicos que, pudimos constatarlo empíricamente, son necesarias para realizar la construcción de narrativas tecnológicas (Laudel y Gläser 2007:101). 
La primera es la creación de un lenguaje ad hoc. Este es uno de los aspectos más importantes. La conducción de la entrevista requiere de un lenguaje que permita al entrevistador obtener información relevante. Este lenguaje debe ser comprensible tanto para entrevistador como entrevistado, y debe facilitar la descripción del mundo del entrevistado.

Para lograr un lenguaje común que dé cuenta de la intersección entre el mundo de la investigación sociológica y el mundo del trabajo del científico, el entrevistador no sólo debe ajustar su lenguaje al trasfondo cultural del entrevistado, sino que crear un lenguaje en el que las experiencias de trabajo relevantes puedan ser descritas de una forma inteligible por las dos partes. En este proceso, el entrevistador debe adoptar elementos del entrevistado y viceversa.

La segunda tarea es la preparación de la entrevista. Conducir una entrevista de este tipo requiere de preparación. El entrevistador tiene, por lo general, escasas oportunidades para recoger bien la información y es difícil que ocurra un proceso de aprendizaje en terreno. De allí que sea necesario un aprendizaje previo al momento de la entrevista. Laudel y Gläser proponen tres estrategias: estudiar textos de referencia de la disciplina, obtener antecedentes acerca del trabajo o investigación del entrevistado (siempre), y analizar publicaciones del entrevistado.

Finalmente, una tercera tarea es la de negociar el nivel de comunicación. Cada entrevista se inicia con una fase implícita de negociación. Parte de esas negociaciones es que el entrevistador sugiere un vocabulario, el cual es modificado a su vez por la respuesta del entrevistado. En esta fase introductoria, mientras se negocian los términos técnicos que puede usar el científico para que sea comprendido adecuadamente por el entrevistador, simultáneamente se negocia cuán científicas deben ser las explicaciones para que éstas sean comprensibles.

La construcción del informe: La elaboración de narrativas tecnológicas requiere de la reconstrucción de la historia de la tecnología.

El levantamiento de información se inició con una primera entrevista, donde se solicitó dar cuenta de la "historia" del caso en general, desde el primer evento o elemento que desencadenará la trayectoria. A partir de esta primera entrevista se solicitaron documentos de segundo orden.

Con esta información, se construyó una primera versión de la trayectoria de la tecnología, la cual toma la forma de viñetas consecutivas ordenadas en lo posible cronológicamente. Cada viñeta daría cuenta de algún elemento, humano o no humano, que se constituyera como parte de la trayectoria.

Una vez realizada la primera versión de la trayectoria se solicitó al interlocutor su revisión. Esta primera versión incluía preguntas para resolver determinados aspectos de la trayectoria. El interlocutor debía, por lo tanto, corregir, especificar y agregar aquello que no se haya inscrito en la primera versión, así como responder las preguntas formuladas. Este trabajo era realizado de forma autónoma por el interlocutor.

A partir de las correcciones de la primera versión, se dio paso a construir una segunda versión, reiniciando el ciclo. De esta forma, la recolección de datos continúa principalmente a través de la retroalimentación de los interlocutores acerca de cada nueva versión de la trayectoria tecnológica. Simultáneamente, se continúa realizando un análisis de documentos de segundo orden. En ocasiones las entrevistas fueron acompañadas de explicaciones verbales y/o gráficas para dar cuenta con mayor claridad los aspectos científicos y técnicos 
del caso. Es relevante destacar que cada nueva retroalimentación y datos recolectados de la documentación implican un re-tratamiento del conjunto de los elementos para reorganizar la historia del objeto técnico.

En promedio en nuestra investigación se realizaron 5 versiones para cada innovación, con un mínimo de 3 versiones y un máximo de 6 versiones. Se determinaba el haber alcanzado la versión final de la trayectoria cuando se consideraba que se habían resuelto todas las preguntas formulables a la trayectoria y cuando el interlocutor lograba enunciar que efectivamente la trayectoria lograba dar cuenta de "cómo ocurrieron las cosas", obteniendo una descripción saturada.

Este procedimiento puede asimilarse a la lógica de comparación constante de la teoría fundamentada de Glasser y Strauss (Vieytes 2004), el que plantea un proceso simultáneo de recolección, codificación y análisis de los datos. La comparación continua permite ir refinando los conceptos, identificando sus propiedades, explorar sus interrelaciones, para integrarlo, finalmente, en un todo coherente. En nuestro caso, este procedimiento permite alcanzar una descripción saturada y coherente de la "historia" de la tecnología. Una vez que se cuenta con la descripción saturada de la trayectoria de la tecnología hasta el momento que ocurre el registro, recordando que la cadena de asociaciones de la innovación permanece en una constante transformación, se da paso a la construcción de las narrativas.

\section{Algunas limitaciones a tener en consideración}

Nuestra investigación tenía como objetivo identificar factores comunes y diferenciadores en las trayectorias de las innovaciones. Para ello se hizo un tratamiento transversal de las siete narrativas a través de la organización de la información en un mapa cognitivo utilizando el software NVIVO.

No obstante, al apegarse a los principios de la sociología de las asociaciones, las narrativas tecnológicas no permitirían ir más allá del estudio de caso: la explicación emerge de la saturación de la red sociotécnica, el que sería producto de un entramado de entidades único e irrepetible. Aun cuando la investigación levantó siete narrativas tecnológicas, bajo estos principios la posibilidad de aplicar una lógica inductiva y eventualmente realizar proposiciones en vista a generalizar estaría descartada.

Por otra parte, Latour advierte que nunca se debe predeterminar lo que es esencial y lo que no. Un segmento particular de un programa de acción no puede ser considerado como la esencia de una innovación y los otros simplemente como el contexto, el embalaje, la historia o el desarrollo. En este sentido, el peso de los factores debe ser "calculado" como una función del movimiento de las cadenas de asociaciones, los que variarán a su vez en cada historia (Latour 1998a:123).

Los elementos que podrían ser identificados como facilitadores u obstaculizadores no pueden ser extraídos del entramado del que forman parte, en la medida que adquieren significado en dicho entramado. Los elementos aislados son, desde esta perspectiva, cuasi-sujetos o cuasi-objetos (7).

En otras palabras, un elemento no podría ser esencialmente ni facilitador ni obstaculizador, sino que "al igual que un vector, pueden tener o presentar una direccionalidad según el momento en que sean descritos, según la ordenación o distribución en que aparezcan" (Domènech y Tirado 1998:29).

En el caso de la investigación, para alcanzar los objetivos planteados, el uso de las narrativas tecnológicas fue formal. A partir de las siete narrativas se aplicó una lógica inductiva, donde se organizaron los factores facilitadores (originados en los programas) y los obstaculizadores (originados en los antiprogramas). 
A partir de esta organización transversal de la información, se describieron los factores que emergían como recurrentes, correspondiendo a los distintos actantes identificados: el innovador (entidad de I\&D), la industria receptora de la tecnología, la institucionalidad, la tecnología y el problema ambiental. A su vez, dado el número relativamente escaso de narrativas, se describieron aquellos factores que emergían con menor recurrencia, pero que eventualmente en el caso de contar con un número mayor de narrativas, éstas podrían haber mostrado mayor recurrencia. De esta forma, a partir del mapa cognitivo, se describieron 25 factores facilitadores y 16 factores obstaculizadores.

No se puede sacar conclusiones a partir de la cantidad de factores identificados positivos o negativos; en algunos casos un elemento obstaculizador muy "poderoso" en el contexto de la narrativa puede anular muchos elementos facilitadores.

En relación con la capacidad descriptiva/ explicativa, estos factores fueron contrastados con los actores y reconocidos por ellos en su experiencia en el desarrollo de las innovaciones.

\section{Acerca de sus potencialidades}

Según Joseph Schumpeter, el desarrollo económico se produce a través de la innovación por medio de un proceso dinámico en el cual las nuevas tecnologías sustituyen a las antiguas (OECD 2006:37). Esta premisa se encuentra hoy en día extendida en todas las agendas políticas, tanto de los países desarrollados como en los países en vías de desarrollo. El estudio acerca de la innovación adquiere por tanto una relevancia significativa más allá del interés académico.

Pinch y Bijker, ambos exponentes de SCOT -Social Construction of Technology- realizan una crítica a los estudios sobre innovación, indicando que éstos han sido desarrollados principalmente por economistas con el propósito de conocer las condiciones de éxito de la innovación. Los factores investigados incluyen sobre todo aspectos macroeconómicos así como la capacidad de innovación de la firma, por ejemplo, el tamaño de los recursos destinados a I\&D, liderazgo y capacidad de marketing, dejando a la tecnología como una caja negra (Pinch y Bijker 1984:404).

La sociología de las asociaciones, por el contrario, provee de un marco conceptual que permite comprender y estudiar los procesos de innovación en sus particularidades, lo que abre una oportunidad para alcanzar una mayor comprensión del proceso de innovación en sí mismo.

Esto puede ser particularmente relevante para el estudio de la innovación en los países en vías de desarrollo.

Estos por lo general, sea desde la teoría del sistema mundo o de la dependencia, sea desde la teoría de la modernización, son cubiertos por premisas y conceptos que toman cuerpo en fuerzas, estructuras, inclusive culturas, para explicar la condición asimétrica de estos países.

Para la sociología de las asociaciones, estas explicaciones vacían de todo significado real a las desigualdades sociales: al dejar de lado los medios prácticos, es decir, los mediadores a través de los cuales se produce la inercia, la durabilidad, la asimetría, la extensión, la dominación, y al fusionar todos esos medios diferentes con el poder impotente de la inercia social, se ocultan las verdaderas causas de las desigualdades sociales (Latour 2008:125). Latour plantea que si hay que luchar con una fuerza que es invisible, no rastreable, ubicua y total, no se tiene poder alguno. Sólo se puede tener alguna posibilidad de modificar un estado 
determinado de cosas si las fuerzas están hechas de vínculos más pequeños, cuya resistencia puede ser probada una a una (Latour 2008:349).

Con esto, la expectativa de alcanzar mayores niveles de desarrollo con base en la innovación, entre otros por supuesto, requiere de una mirada aguzada que permita hacer frente a los antiprogramas que emergen en los caminos, tanto locales como globales, expresados todos como mediadores en un mismo plano.

Estudios de este tipo permitirían conocer con mayor profundidad cómo ocurren los procesos de innovación a nivel local, diferenciándose de cómo estos ocurren en los países altamente industrializados, desde una perspectiva que focaliza, más que en las restricciones económicas y estructurales, en identificar las entidades relevantes locales que participan en los entramados de las innovaciones propias.

Esta metodología también se puede extender a los estudios sobre transferencia de tecnología, procesos habituales en países en vías de desarrollo, ampliamente reconocidos por su gran complejidad y que presentan, por lo general, altas tasas de fracaso (Ruffier 1998, Villavicencio y Arvanitis 1996, Levin 1997). Los objetos técnicos suelen ser reinventados por los utilizadores cuando la tecnología es exógena (Akrich 2006). En estos casos, el entramado de la innovación inicia una nueva dinámica de traducción, de movilización de entidades, para lograr estabilizar la tecnología en el nuevo contexto, por lo que la aproximación simétrica puede aplicarse nuevamente, develando las condiciones específicas en torno a estos procesos.

\section{Conclusiones}

Hemos expuesto en este artículo una breve síntesis de las bases conceptuales de la sociología de las asociaciones y una metodología asociada, aplicada particularmente a los estudios de la innovación.

No obstante, el alcance de sus planteamientos trasciende su campo original de desarrollo, por cuanto se propone como una nueva forma de hacer sociología. Esto implica la necesidad de explorar y comprobar su eficacia en otros ámbitos y objetos de estudio.

En el caso de nuestra investigación, la utilización de este método permitió generar hallazgos de sumo interés, que iban más allá de las causas sociales que se esperaban encontrar. De esta forma, además de identificar aspectos organizacionales relevantes tanto en el proveedor de tecnología, en el receptor de la tecnología, como en la institucionalidad pública y sus condiciones para proveer de subsidios a estos desarrollos; además de identificar algunas lógicas de mercado que juegan un rol crucial al momento de escalar las innovaciones y lograr estabilizarlas, se descubrió la relevancia del proceso de selección de la tecnología y su compatibilidad con el problema ambiental localizado como un elemento clave; la versatilidad y capacidad de la tecnología para adaptarse a los cambios productivos o contingencias, los tiempos de desarrollo y proceso científico de desarrollo de la innovación, entre otros procesos donde entidades de materia no social jugaban un rol altamente significativo.

Explorar en nuevos objetos de estudio que den cuenta empíricamente de la potencialidad explicativa de esta nueva aproximación se abre como un campo de investigación desafiante y relevante, sobre todo ante la posibilidad de ampliar efectivamente la capacidad heurística de nuestra disciplina. 


\section{Notas}

Esta investigación fue realizada con fondos de la Facultad de Ciencias Sociales de la Universidad de Concepción (Concepción, Chile).

(1) En inglés Actor-Network-Theory, conocida también por su abreviación ANT.

(2) Desde otra perspectiva Barriga y Henríquez (2005) definen lo social como subjetividad compartida.

(3) Este es el significado técnico de la palabra "red". Se puede definir como una serie de acciones en la que cada participante es tratado como un mediador (Latour 2008:187). Es una herramienta para ayudar a describir algo. Una red no es lo que se está representando en el texto, sino lo que prepara al texto para tomar la posta de los actores como mediadores (Latour 2008:190).

(4) A esto Latour se refiere como la primera fuente de incertidumbre, señalando que no existen grupos sino que sólo formación de grupos. La estabilización de lo social es tarea de los actores, no de los analistas (Latour 2008:47).

(5) Esto no es casual. El origen de la Teoría del Actor-Red se debe a la confrontación de las explicaciones sociales frente a científicos, donde no fue posible legitimar fuerzas sociales como causas para producir como efectos hechos objetivos. Ver más en Latour (2008:138).

(6) Para conocer con mayor detalle la definición de “innovación para el mundo" e “innovación para el mercado" ver OECD (2006).

(7) Para conocer en profundidad los conceptos de cuasi-objeto y cuasi-sujeto, ver los trabajos de Serres (1991).

\section{Bibliografía}

Akrich, M. 2006. Les objets techniques et leurs utilisateurs. De la conception à l'action. En: M. Akrich, M. Callon y B. Latour (eds). Sociologie de la Traduction. Textes fondateurs. Paris: Mines Paris Les Presses, pp. 179- 200.

Barriga, O. y Henríquez, G. 2005. El plano alfa del objeto de estudio. Cinta moebio 24: 1-15.

Callon, M. 1998. El proceso de la construcción de la sociedad. El estudio de la tecnología como herramienta para el análisis sociológico. En: M. Domènech y F.J. Tirado (comps.). Sociología simétrica. Ensayos sobre ciencia, tecnología y sociedad. Barcelona: Gedisa Editorial, pp.143- 170.

Domènech, M. y Tirado, F. J. 1998. Sociología simétrica. Ensayos sobre ciencia, tecnología y sociedad. Barcelona: Gedisa Editorial.

Latour, B. 1998a. La tecnología es la sociedad hecha para que dure. En: M. Domènech y F. J. Tirado (comps.). Sociología simétrica. Ensayos sobre ciencia, tecnología y sociedad. Barcelona: Gedisa Editorial, pp. 109-142. 
Latour, B. 1998b. De la mediación técnica: filosofía, sociología, genealogía. En: M. Domènech y F. J. Tirado (comps.). Sociología simétrica. Ensayos sobre ciencia, tecnología y sociedad. Barcelona: Gedisa Editorial, pp. 249- 302.

Latour, B. 2008. Reensamblar lo social. Una introducción a la teoría del actor-red. Buenos Aires: Ediciones Manantial.

Laudel, G. y Gläser, J. 2007. Interviewing scientists. Science, Technology \& Innovation Studies 3: 91-111.

Levin, M. 1997. Technology transfer is organizational development: An investigation into the relationship between technology transfer and organizational change. International Journal of Technology Management 14: 297- 308.

Meyer, U. y Schubert, C. 2007. Integrating path dependency and path creation in a general understanding of path constitution. The role of agency and institutions in the stabilization of technological innovations. Science, Technology and Innovations Studies 3: 23-44.

OECD. 2006. Manual de Oslo. Guía para la recogida e interpretación de datos sobre innovación. Madrid: Tragsa Ediciones.

Pinch, T. y Bijker, W. 1984. The social construction of facts and artefacts: or How the Sociology of Science and the Sociology of Technology might benefit each other. Social Studies of Science 14: 399-441.

Ruffier, J. 1998. La eficiencia productiva: cómo funcionan las fábricas. Montevideo: Cinterfor.

Serres, M. 1991. Historia de las ciencias. España: Cátedra.

Vieytes, R. 2004. Metodología de la investigación en organizaciones, mercado y sociedad. Epistemología y técnicas. Buenos Aires: Editorial de las Ciencias.

Villavicencio, D. y Arvanitis, R. 1996. Transferencia de tecnología y aprendizaje tecnológico: reflexiones basadas en trabajos empíricos. El Trimestre Económico 16: 257-279.

Recibido el 18 Ene 2011

Aceptado el 20 Jun 2011 